\title{
Relationship of Serum Soluble Klotho Levels and Echocardiographic Parameters in Patients on Maintenance Hemodialysis
}

\author{
Ai-hua Zhang Wei-kang Guo Ling Yu Wen-hu Liu \\ Department of Nephrology, Affiliated Beijing Friendship Hospital, Nephrology Faculty, \\ Capital Medical University, Beijing, PR China
}

\section{Keywords}

Soluble Klotho · Interventricular septal thickness · Uremic cardiomyopathy

\begin{abstract}
Background: Cardiovascular disease is the leading cause of morbidity and mortality in maintenance hemodialysis (MHD) patients. Uremic cardiomyopathy, characterized by myocardial hypertrophy and fibrosis, has a significant contribution to these adverse cardiac outcomes. The protective effect of soluble Klotho (s-Klotho) on myocardial damage was demonstrated in in vitro and animal experiments. However, data from MHD patients is limited. The present study was designed to identify potential correlations between echocardiographic parameters and serum s-Klotho levels in MHD patients. Methods: This is a cross-sectional study involving 105 MHD patients from the Dialysis Center of Capital Medical University affiliated Beijing Friendship Hospital between March and October 2014. The general information for each patient was recorded. Fasting blood samples were collected prior to hemodialysis during the mid-week session in all patients. The echocardiogram and left lateral lumbar spine radiograph were performed after the same mid-week session. The dialysis records for each session within 3 months before the blood tests were documented. According to the quartiles of s-Klotho levels, patients were divided into four groups (Group 1-4). The demographic and clinical characteristics, echocardiographic parameters, and abdominal aortic calcification scores among the groups were compared. Results: The enrolled 105 patients were predominantly male (54.3\%) with an average age of $59.9 \pm 11.2$ years. Previous hemodialysis durations were 76 (42-133) months. Sixteen (15.2\%) patients had diabetes mellitus. Mean serum s-Klotho level
\end{abstract}




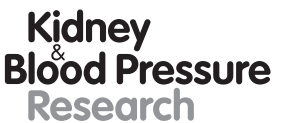

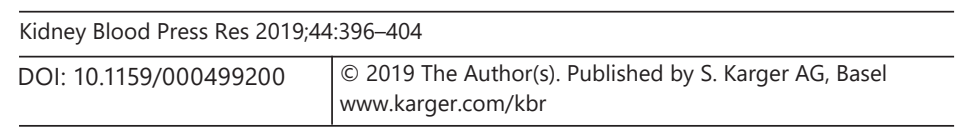

Zhang et al.: S-Klotho and Echocardiographic Parameters

was $411.83 \pm 152.95 \mathrm{pg} / \mathrm{mL}$, and the 25 th percentile, 50th percentile, and 75 th percentile values of serum s-Klotho levels were $298.9,412$, and $498.2 \mathrm{pg} / \mathrm{mL}$, respectively. Individuals in the bottom quartile of s-Klotho levels (Group 1) had significantly increased interventricular septal thickness (IVST) compared to those in the other three quartiles of s-Klotho levels (Group 1: $1.12 \pm 0.16 \mathrm{~cm}$; vs. Group 2: $1.12 \pm 0.16 \mathrm{~cm}, p=0.008$; vs. Group 3: $0.94 \pm 0.13 \mathrm{~cm}, p<0.001$; vs. Group 4: $1.03 \pm 0.15 \mathrm{~cm}, p=0.022$ ). There were significant differences in the ratios of IVST and posterior wall thickness (PWT) between patients of Group 1 and Group 3 (1.12 \pm 0.12 vs. $1.00 \pm 0.14, p=0.004)$. No significant differences were found for other parameters among the groups. The univariate correlation analyses showed that gender $(r=-0.211, p=0.030)$, $\mathrm{Kt} / \mathrm{V}$ urea $(r=-0.240, p=0.014)$, hypersensitive $C$ reactive protein (hs-CRP) $(r=0.196, p=$ $0.045)$, and serum s-Klotho levels $(r=-0.260, p=0.007)$ significantly correlated with IVST. UItimately, only hs-CRP and serum s-Klotho levels were entered into a multiple regression model. Conclusions: The present study showed that patients with lower circulating s-Klotho levels were more often associated with larger IVST and greater ratios of IVST and PWT. There was an independent association between s-Klotho and IVST, and lower s-Klotho levels seem to be a potential risk factor of uremic cardiomyopathy in MHD patients.

(C) 2019 The Author(s)

Published by S. Karger AG, Basel

\section{Introduction}

Cardiovascular disease is the leading cause of morbidity and mortality in maintenance hemodialysis (MHD) patients [1]. Chronic kidney disease (CKD)-related cardiomyopathy, which has been termed "uremic cardiomyopathy" [2], has a significant contribution to these adverse cardiac outcomes [3]. Clinically, uremic cardiomyopathy is characterized by cardiac arrest or sudden death, left ventricular hypertrophy, cardiac fibrosis, congestive heart failure, and so on [4-6]. In addition to traditional risk factors such as smoking, hypertension, diabetes, anemia, and volume overload, Klotho deficiency also plays an important role because soluble Klotho (s-Klotho) is a circulating substance exerting multiple systemic biological actions on distant organs, such as directly protecting cells against a variety of insults including hypoxia, hyperoxia, oxidative stress, and cytotoxic medication and suppressing apoptosis $[6,7]$.

However, the available evidence about the relationship of s-Klotho and uremic cardiomyopathy are limited [8]. Hu et al. [8] had observed left ventricular hypertrophy in Klotho-deficient animals. Kim and his colleagues [9] analyzed 2,101 CKD patients (the mean estimated glomerular filtration rate was $53.0 \pm 30.7 \mathrm{~mL} / \mathrm{min} / 1.73 \mathrm{~m}^{2}$ ) and reported that serum Klotho was an independent biomarker of left ventricular mass index (LVMI). But another crosssectional study with 88 MHD patients showed that s-Klotho was significantly associated with left ventricular ejection fraction (LVEF), but not with LVMI [10].

In light of these conflicting data, the present study was designed to identify potential correlations between echocardiographic parameters and serum s-Klotho levels in MHD patients.

\section{Methods}

\section{Study Population}

This is a cross-sectional study involving 105 MHD patients from the Dialysis Center of Capital Medical University affiliated Beijing Friendship Hospital between March and October 2014. All patients were stable on thrice-weekly dialysis therapy for at least 6 months before the start of the study, had a permanent arteriovenous access, and were aged 18 to 75 years. 


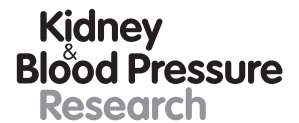

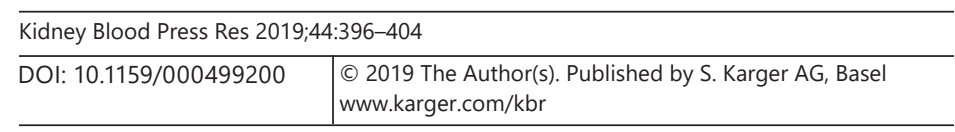

Zhang et al.: S-Klotho and Echocardiographic Parameters

High-flux polysulfone, polyethersulfone, or polymethylmethacrylate membrane dialyzers were used with a membrane area of 1.5-1.8 $\mathrm{m}^{2}$, and blood flow was between 220 and 300 $\mathrm{mL} / \mathrm{min}$. Ultrapure dialysis fluid (bacteria $<0.1 \mathrm{CFU} / \mathrm{mL}$ and endotoxin $<0.03 \mathrm{IU} / \mathrm{mL}$ ) was used. The composition of the dialysis fluid was identical: bicarbonate, $35 \mathrm{mmol} / \mathrm{L}$; acetate, 3.7 $\mathrm{mmol} / \mathrm{L}$; sodium, $138 \mathrm{mmol} / \mathrm{L}$; calcium, $1.75 \mathrm{mmol} / \mathrm{L}$; magnesium, $0.5 \mathrm{mmol} / \mathrm{L}$; potassium $3.0 \mathrm{mmol} / \mathrm{L}$; chloride, $110 \mathrm{mmol} / \mathrm{L}$. Dialysate flow was $500 \mathrm{~mL} / \mathrm{min}$. Dialysate temperature was constantly kept at $36.5^{\circ} \mathrm{C}$. Anticoagulation was achieved by heparin or low-molecularweight heparin. Kt/V was not less than 1.2, while residual glomerular filtration rate was less than $5 \mathrm{~mL} / \mathrm{min}$.

Patients were excluded from the study if they had severe heart failure (New York Heart Association Class III or IV), primary cardiomyopathy, malignancy, autoimmune disease, evidence of acute or chronic infection, and a history of renal transplantation or peritoneal dialysis. Informed written consent was obtained from all participants, and the study protocol was approved by the institute's ethics committee.

The general information for each patient was recorded. Fasting blood samples were collected prior to hemodialysis during the mid-week session in all patients. Routine blood tests were performed immediately including hemoglobin, creatinine, albumin, hypersensitive $\mathrm{C}$ reactive protein (hs-CRP), calcium (corrected), phosphate, and intact parathyroid hormone values. Corrected calcium levels were calculated using the following formula [11]: corrected calcium $=$ calcium $+0.8 \times(4-$ serum albumin $)$. Blood samples for $\mathrm{s}$-Klotho measurement were centrifuged, aliquoted in vials, and stored at $-80^{\circ} \mathrm{C}$ until assay. According to the quartiles of s-Klotho levels, patients were divided into four groups. The echocardiogram and left lateral lumbar spine radiograph were performed after the same mid-week session. The dialysis records for each session within 3 months before the blood tests were documented.

\section{Serum Levels of s-Klotho Measurement}

Serum s-Klotho levels were determined using a sandwich enzyme-linked immunosorbent assay (ELISA) kit according to the manufacturer's protocol (ImmunoBiological Laboratories Co., Ltd, Tokyo, Japan). This assay detects circulating s-Klotho by using two monoclonal antibodies that specifically recognize the extracellular domain of Klotho [12]. Means \pm standard deviation (SD) were $6.5 \pm 3.3 \%$ for inter-assay and $3.6 \pm 1.5 \%$ for intra-assay coefficients of variability [13].

\section{Echocardiographic Examination}

Echocardiographic examination was performed using a Philips iE33 system with a multifrequency transducer and harmonic imaging as appropriate. A two-dimensional guided M-mode measurement and conventional pulse wave Doppler were performed according to the American Society of Echocardiography guidelines [14]. Left atrial diameter, left ventricular end-diastolic volume (LVEDD), left ventricular posterior wall thickness (PWT), interventricular septal thickness (IVST), and LVEF were determined. Left ventricular mass (LVM) was estimated using the anatomically validated formula of Devereux et al. [15] and was indexed to height ${ }^{2.7}$ (LVMI): LVM [g] $=0.8 \times\left(1.04 \times(\text { LVEDD + PWT + IVST })^{3}-(\text { LVEDD })^{3}\right)+0.6[16]$.

\section{Lateral Lumbar Radiography and Evaluation of Abdominal Aortic Calcification}

Lateral lumbar X-ray was performed in a standing position using standard radiographic equipment. A minimum of $8 \mathrm{~cm}$ of tissues anterior to the lumbar spine, which would include abdominal aorta, had to be visible: the focus-film distance was $100 \mathrm{~cm}$. Other parameters were: $90 \mathrm{kV}, 33-200 \mathrm{mAs}$ (depending on the body habitus), and the estimated dose of radiation was approximately $15 \mathrm{mGy}$. 


\section{Kidney \\ Blood Pressure \\ Research}

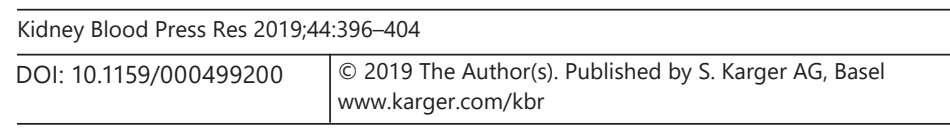

Zhang et al.: S-Klotho and Echocardiographic Parameters

Abdominal aortic calcification (AAC) was assessed using a previously validated 24-point scale [17]. All subjects were assessed independently by two investigators blinded to patient demographics and serum markers. For the 24-point score, calcified deposits along the anterior and posterior longitudinal walls of the abdominal aorta adjacent to each lumbar vertebra from L1 to L4 were assessed using the midpoint of the intervertebral space above and below the vertebrae as the boundaries. Calcifications were graded as follows: 0 , no aortic calcific deposits; 1 , small scattered calcific deposits less than one-third of the corresponding length of the vertebral levels; 2 , medium quantity of calcific deposits about one-third or more, but less than two-thirds of the corresponding vertebral length; 3 , severe quantity of calcifications of more than two-thirds or more of the corresponding vertebral lengths. The scores, obtained separately for the anterior and posterior walls, result in a range from 0 to 6 for each vertebral levels and 0 to 24 for the total score.

\section{Blood Pressure Measurement}

Systolic blood pressure (SBP) and diastolic blood pressure (DBP) were measured predialysis (pre-SBP and pre-DBP) and $15 \mathrm{~min}$ after the end of dialysis (post-SBP and post-DBP) using the Omron HEM-4030 electronic sphygmomanometer in the non-fistula side of the upper arm. All these values were collected over the 3 months before the blood samples were obtained and time-averaged.

\section{Statistical Analysis}

Results are expressed as mean \pm SD or median and interquartile range, depending on data distribution, for continuous variables and as frequency for categorical variables. Statistics were carried out using the software SPSS 11.5 for Windows (SPSS, Inc., Chicago, IL, USA). For comparison among the groups, ANOVA analysis was used for normal-distributed continuous variables and the nonparametric test for nonnormal-distributed ones, while the $\chi^{2}$ test was employed for categorical variables. Pearson or Spearman correlation was used to analyze the association between demographic, laboratory, blood pressure, or AAC data and echocardiographic parameters. Significant variables were furthermore identified by stepwise linear regression. $p$ less than 0.05 was considered statistically significant.

\section{Results}

\section{Patient Characteristics}

The enrolled 105 patients were predominantly male (54.3\%) with an average age of 59.9 \pm 11.2 years. Previous hemodialysis durations were 76 (42-133) months. Sixteen $(15.2 \%)$ patients had diabetes mellitus. Mean serum s-Klotho levels were $411.83 \pm 152.95 \mathrm{pg} / \mathrm{mL}$, and the 25 th percentile, 50 th percentile, and 75 th percentile values of serum s-Klotho levels were $298.9,412$, and $498.2 \mathrm{pg} / \mathrm{mL}$, respectively. Patients were divided into 4 groups according to the quartiles: Group 1: s-Klotho $\leq 25$ th percentile $(n=26$, mean s-Klotho: $224.0 \pm 47.8 \mathrm{pg} /$ $\mathrm{mL})$; Group 2: 25 th percentile $<$ s-Klotho $\leq 50$ th percentile $(n=27$, mean s-Klotho: $366.4 \pm$ $30.9 \mathrm{pg} / \mathrm{mL})$; Group 3: 50 th percentile $<\mathrm{s}$-Klotho $\leq 75$ th percentile $(n=26$, mean s-Klotho: $455.5 \pm 25.2 \mathrm{pg} / \mathrm{mL})$; Group 4: s-Klotho $\geq 75$ th percentile $(n=26$, mean s-Klotho: $613.1 \pm$ $105.8 \mathrm{pg} / \mathrm{mL}$ ) (Table 1$)$.

\section{Comparison among the Groups}

There were significant differences in IVST and the ratios of IVST and PWT among the groups (Table 1). Post hoc multiple comparisons showed that individuals in the bottom quartile of s-Klotho levels (Group 1) had significantly increased IVST compared to those in 


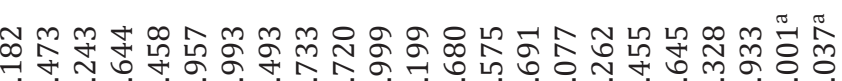

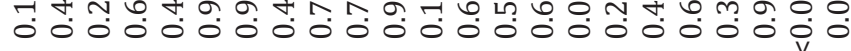

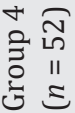

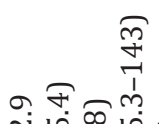

เก

ำ

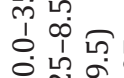

겅

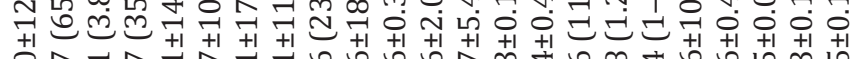

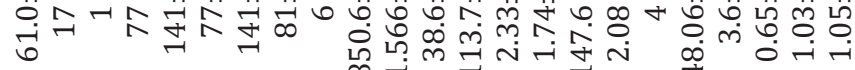

mo

ङ

언

สุกฺฺ

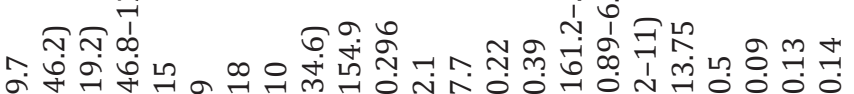
$+1=0$

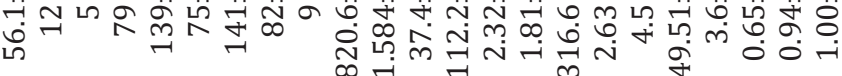

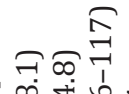<smiles>C1CCCC1</smiles> \\ (1)} ง $\underset{4}{4}$

\section{i্ \\ तै}

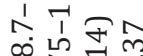

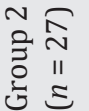
+

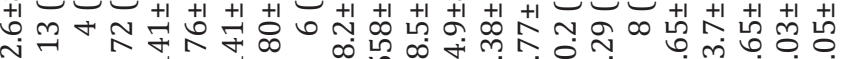

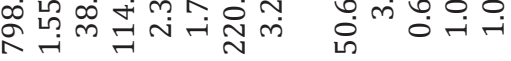

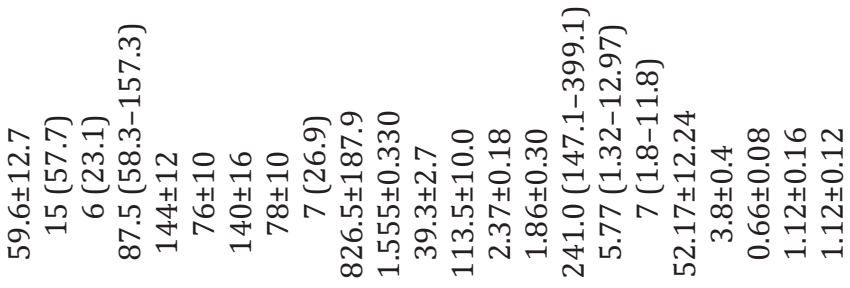

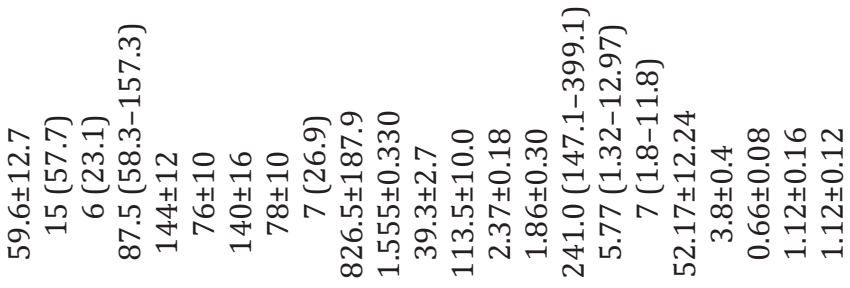

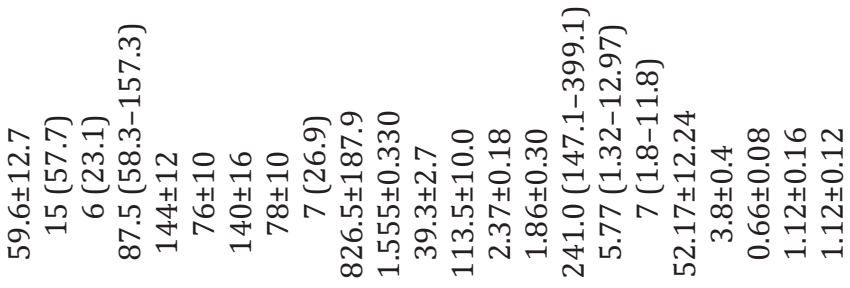

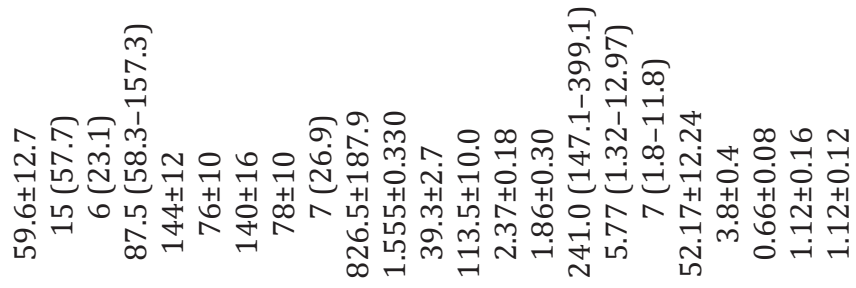

总

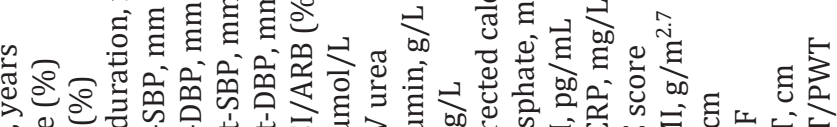

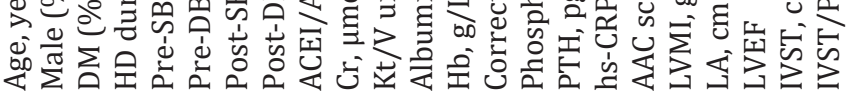<smiles>CCCCCCCCCCCC</smiles>

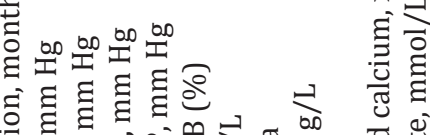

苟 
Table 2. Univariate correlation analyses of factors associated with IVST

the other three quartiles of s-Klotho levels (Group 1: $1.12 \pm 0.16 \mathrm{~cm}$; vs. Group 2: $1.12 \pm 0.16$ cm, $p=0.008$; vs. Group 3: $0.94 \pm 0.13 \mathrm{~cm}, p<0.001$; vs. Group 4: $1.03 \pm 0.15 \mathrm{~cm}, p=0.022$ ). As to the ratios of IVST and PWT, the differences mainly existed in between patients of Group 1 and Group 3 ( $1.12 \pm 0.12$ vs. $1.00 \pm 0.14, p=0.004)$. No significant differences were found for other parameters among the groups.

Predictive Values of Serum s-Klotho for Interventricular Septal Thickness

Firstly, the univariate correlation analyses were performed to identify potential risk factors of IVST. Gender $(r=-0.211, p=0.030)$, Kt/V urea $(r=-0.240, p=0.014)$, hs-CRP $(r=$ $0.196, p=0.045)$, and serum s-Klotho levels $(r=-0.260, p=0.007)$ significantly correlated with IVST (Table 2). Ultimately, two independent variables were entered into a multiple regression model, which were hs-CRP and serum s-Klotho levels. The model explained 12.9\% of the variability in IVST $(p<0.001)$. Hs-CRP and serum s-Klotho levels emerged as independent predictors of IVST, with contribution to the variation in IVST being 10.2 and $2.7 \%$, respectively; their standardized coefficients were $0.288(p=0.003)$ and $-0.193(p=0.043)$, respectively.

\section{Discussion}

In the present cross-sectional study, we showed that patients with lower serum s-Klotho levels had larger IVST and greater ratios of IVST and PWT, and that serum s-Klotho level was independently and inversely associated with IVST.

Circulating s-Klotho is produced either by proteolytic cleavage of the extracellular domain of the transmembrane form or by alternative mRNA splicing $[18,19]$, which exerts pleiotropic actions independently of FGF-23 in an autocrine, paracrine, and endocrine fashion [20]. Growing evidence showed that Klotho deficiency plays an important role in myocardial 


\section{Kidney \\ Blood Pressure \\ Research}

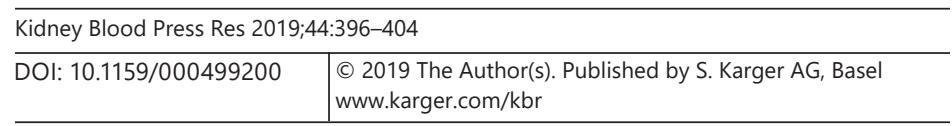

Zhang et al.: S-Klotho and Echocardiographic Parameters

hypertrophy, fibrosis, and remodeling. Both primary genetic Klotho deficiency (heterozygous Klotho-deficient, kl/+ mice) and secondary Klotho deficiency (from phosphate loading, aging, and CKD) triggered cardiac hypertrophy and fibrosis in mice [21, 22]. Hu et al. [21] observed that lower plasma Klotho levels were associated with more severe cardiac hypertrophy and fibrosis. Left ventricular hypertrophy persisted in Klotho deficiency CKD animals after correcting hyperphosphatemia due to dietary phosphate restriction and consequently reducing FGF-23 levels, indicating an independently protective effect from Klotho [21, 22]. Moreover, Klotho replacement was found to reduce isoproterenol-induced cardiomyocyte apoptosis [23], prevent left ventricular hypertrophy in CKD mice, and block cardiomyocyte hypertrophy in vitro [24]. However, data from CKD, especially uremic patients, is limited. Kim et al. [9] analyzed 2,101 participants with CKD from stage 1 to 5 (pre-dialysis). A multivariable linear regression model showed serum Klotho had a significant inverse association with LVMI. But in a more recent cross-sectional study including 88 patients with end-stage renal disease on regular hemodialysis, s-Klotho showed significant association with LVEF, but not with LVM and LVMI [10]. Similarly, Buiten et al. [25] also failed to find a relationship between serum s-Klotho and LVMI in 127 dialysis patients. Our results are in agreement with those of the latter two studies from dialysis patients. LVMI was not significantly different among varying groups of serum s-Klotho levels. A possible explanation is that LVMI of dialysis patients are affected by more factors than non-dialysis patients with CKD, such as interdialytic weight gain, ultrafiltration, dialysate, dialyzer, and so on.

Additionally, our results illustrated that lower serum s-Klotho levels are related to larger IVST and greater ratios of IVST and PWT in MHD patients. Serum s-Klotho levels are independently and inversely associated with IVST. These were not investigated before. The dimension of differences among the groups seemingly was small, but it has clinical significance considering the fact that the normal thickness of the LV myocardium is 6 to $9 \mathrm{~mm}$ in women and 6 to $10 \mathrm{~mm}$ in men [26]. Although the prognostic value of IVST has some controversy, the parameter has received more and more attention in some specific populations in recent years. Eliakim-Raz et al. [27] reported an association between IVS thickening and future development of hypertension in a young healthy cohort of 500 Israeli Air Force aviators and argued that IVS thickening may not merely be a result of long-term BP elevation, but may predict the development of systolic hypertension. In a study of hypertensive patients receiving regular treatment, multivariate adjusted analysis showed that greater IVST at baseline was associated with worse cardiovascular outcomes during 5 years of follow-up [28]. Huang et al. [29] concluded that thickened IVS can be served as a reliable marker for predicting all-cause death in Chinese patients with coronary artery disease, even in those with normal LVM. Graham-Brown et al. [30] compared global and segmental native myocardial T1 time of 35 hemodialysis patients and 22 control individuals and found that the median native global T1 time was significantly higher in the hemodialysis group (1,270 vs. 1,085 ms), with the septal regions of hemodialysis patients having significantly higher median T1 times than nonseptal regions $(1,293$ vs. $1,252 \mathrm{~ms})$. Native T1 mapping is a novel cardiac magnetic resonance imaging technique that measures native myocardial $\mathrm{T} 1$ relaxation, a surrogate of myocardial fibrosis, so they suggested that the interventricular septum appeared to be particularly prone to the development of fibrosis in hemodialysis patients. In summary, our findings suggested that IVST might be a useful indicator for uremic cardiomyopathy and poor prognosis in MHD patients.

It has been confirmed in MHD patients that serum s-Klotho levels is independently associated with AAC [31] and coronary artery calcification [32]. In the present study, this tendency was not found, which may be related to the difference of s-Klotho cut-off points and our relatively small sample size. However, when the contributions to IVST were investigated, s-Klotho, rather than AAC scores, entered the final equation. The result furthermore indicates the inde- 
pendent impact of s-Klotho on IVST. Some in vitro and animal experiments support that $\mathrm{s}$-Klotho is a direct modulator of pathological cardiac remodeling. Hu et al. and Xie et al. [21, $22]$ demonstrated that Klotho blocked TGF- $\beta 1$ and angiotensin II-induced hypertrophy and fibrosis in cardiomyocytes. Yang et al. [24] reported that the protective effect of Klotho was also achieved by blocking oxidative stress and inhibiting the ERK 1/2 signaling pathway.

In the present study, multivariate linear regression showed that hs-CRP was also correlated to IVST, which is the same as in previous studies [33, 34]. Additionally, s-Klotho is involved in the regulation of the signaling of many cytokines and growth factors. Klotho protein can inhibit insulin/insulin-like growth factor (IGF1) signaling and in this way promote resistance to oxidative stress [18]. In cultured HUVECs, incubation with Klotho results in suppression of expression of cell adhesion molecules, attenuates the activation of NF- $\mathrm{KB}$, and blocks tumor necrosis factor- $\alpha$-induced monocyte adhesion $[35,36]$. Klotho deficiency might be involved in microinflammatory state in MHD patients, and the correlation of hs-CRP and IVST may be partly attributed to Klotho deficiency. Further research is needed.

Our study has some limitations. First, this was a single-center study with a relatively small sample size. Second, data on phosphate binders, active vitamin D, and cinacalcet were not collected, which may affect the relationship among serum s-Klotho, corrected calcium, phosphate, and intact parathyroid hormone levels. Finally, although Klotho has biological actions independently of FGF-23, the confounding effects of FGF-23 cannot entirely be excluded, which was not tested in our study.

In conclusion, the present study showed that patients with lower circulating s-Klotho levels were more often associated with larger IVST and greater ratios of IVST and PWT. There was an independent association between s-Klotho and IVST, and lower s-Klotho levels seem to be a potential risk factor of uremic cardiomyopathy in MHD patients.

\section{References}

1 Bansal N. Evolution of Cardiovascular Disease During the Transition to End-Stage Renal Disease. Semin Nephrol. 2017 Mar;37(2):120-31.

2 de Albuquerque Suassuna PG, Sanders-Pinheiro H, de Paula RB. Uremic Cardiomyopathy: A New Piece in the Chronic Kidney Disease-Mineral and Bone Disorder Puzzle. Front Med (Lausanne). 2018 Jul;5:206.

3 Cerasola G, Nardi E, Palermo A, Mulè G, Cottone S. Epidemiology and pathophysiology of left ventricular abnormalities in chronic kidney disease: a review. J Nephrol. 2011 Jan-Feb;24(1):1-10.

4 London GM, Marchais SJ, Guerin AP, Métivier F. Contributive factors to cardiovascular hypertrophy in renal failure. Am J Hypertens. 1989 Nov;2(11 Pt 2):261S-3S.

5 Guérin AP, Pannier B, Marchais SJ, London GM. Cardiovascular disease in the dialysis population: prognostic significance of arterial disorders. Curr Opin Nephrol Hypertens. 2006 Mar;15(2):105-10.

6 Neyra JA, Hu MC. $\alpha$ Klotho and Chronic Kidney Disease. Vitam Horm. 2016;101:257-310.

7 Lu X, Hu MC. Klotho/FGF23 Axis in Chronic Kidney Disease and Cardiovascular Disease. Kidney Dis (Basel). 2017 Jul;3(1):15-23.

8 Hu MC, Kuro-o M, Moe OW. Secreted klotho and chronic kidney disease. Adv Exp Med Biol. 2012;728:126-57.

9 Kim HJ, Kang E, Oh YK, Kim YH, Han SH, Yoo TH, et al. The association between soluble klotho and cardiovascular parameters in chronic kidney disease: results from the KNOW-CKD study. BMC Nephrol. 2018 Mar; 19(1):51.

10 Abdallah E, Mosbah 0, Khalifa G, Metwaly A, El-Bendary O. Assessment of the relationship between serum soluble Klotho and carotid intima-media thickness and left ventricular dysfunction in hemodialysis patients. Kidney Res Clin Pract. 2016 Mar;35(1):42-9.

11 Kwon HY, Lee OH, Kim MJ, Joo WC, Lee SY, Kim MJ, et al. The association between mortality and abdominal aortic calcification and relation between its progression and serum calcium concentration in chronic hemodialysis patients. Kidney Res Clin Pract. 2014 Jun;33(2):95-102.

12 Pavik I, Jaeger P, Ebner L, Wagner CA, Petzold K, Spichtig D, et al. Secreted Klotho and FGF23 in chronic kidney disease Stage 1 to 5: a sequence suggested from a cross-sectional study. Nephrol Dial Transplant. 2013 Feb; 28(2):352-9.

13 Yu L, Kang L, Ren XZ, Diao ZL, Liu WH. Circulating $\alpha$-Klotho Levels in Hemodialysis Patients and Their Relationship to Atherosclerosis. Kidney Blood Press Res. 2018;43(4):1174-82. 
Zhang et al.: S-Klotho and Echocardiographic Parameters

14 Tranæus Lindblad Y, Olauson H, Vavilis G, Hammar U, Herthelius M, Axelsson J, et al. The FGF23-Klotho axis and cardiac tissue Doppler imaging in pediatric chronic kidney disease-a prospective cohort study. Pediatr Nephrol. 2018 Jan;33(1):147-57.

15 Devereux RB, Dahlöf B, Gerdts E, Boman K, Nieminen MS, Papademetriou V, et al. Regression of hypertensive left ventricular hypertrophy by losartan compared with atenolol: the Losartan Intervention for Endpoint Reduction in Hypertension (LIFE) trial. Circulation. 2004 Sep;110(11):1456-62.

16 Poniku A, Bajraktari G, Elezi S, Ibrahimi P, Henein MY. Adiponectin correlates with body mass index and to a lesser extent with left ventricular mass in dialysis patients. Cardiol J. 2018;25(4):501-11.

17 Toussaint ND, Pedagogos E, Lau KK, Heinze S, Becker GJ, Beavis J, et al. Lateral lumbar X-ray assessment of abdominal aortic calcification in Australian haemodialysis patients. Nephrology (Carlton). 2011 May;16(4): 389-95.

18 Gołembiewska E, Stępniewska J, Kabat-Koperska J, Kędzierska K, Domański M, Ciechanowski K. The role of Klotho protein in chronic kidney disease: studies in animals and humans. Curr Protein Pept Sci. 2016;17(8): 821-6.

19 Chen CD, Tung TY, Liang J, Zeldich E, Tucker Zhou TB, Turk BE, et al. Identification of cleavage sites leading to the shed form of the anti-aging protein klotho. Biochemistry. 2014 Sep;53(34):5579-87.

20 Erben RG. Update on FGF23 and Klotho signaling. Mol Cell Endocrinol. 2016 Sep;432:56-65.

21 Hu MC, Shi M, Cho HJ, Adams-Huet B, Paek J, Hill K, et al. Klotho and phosphate are modulators of pathologic uremic cardiac remodeling. J Am Soc Nephrol. 2015 Jun;26(6):1290-302.

22 Xie J, Yoon J, An SW, Kuro-o M, Huang CL. Soluble klotho protects against uremic cardiomyopathy independently of fibroblast growth factor 23 and phosphate. J Am Soc Nephrol. 2015 May;26(5):1150-60.

23 Song S, Gao P, Xiao H, Xu Y, Si LY. Klotho suppresses cardiomyocyte apoptosis in mice with stress-induced cardiac injury via downregulation of endoplasmic reticulum stress. PLoS One. 2013 Dec;8(12):e82968.

24 Yang K, Wang C, Nie L, Zhao X, Gu J, Guan X, et al. Klotho protects against indoxyl sulphate-induced myocardial hypertrophy. J Am Soc Nephrol. 2015 Oct;26(10):2434-46.

25 Buiten MS, de Bie MK, Bouma-de Krijger A, van Dam B, Dekker FW, Jukema JW, et al. Soluble Klotho is not independently associated with cardiovascular disease in a population of dialysis patients. BMC Nephrol. 2014 Dec;15(1):197.

26 Hudson CC, Hudson JK. The interventricular septum: measurement and motion. Anesth Analg. 2013 Apr; 116(4):788-92.

27 Eliakim-Raz N, Prokupetz A, Gordon B, Shochat T, Grossman A. Interventricular Septum and Posterior Wall Thickness Are Associated With Higher Systolic Blood Pressure. J Clin Hypertens (Greenwich). 2016 Jul;18(7): 703-6.

28 Djordjević D, Tasić I, Stamenković B. Predictive value of non-invasive parameters in patients with left ventricular hypertrophy during a five-year follow-up period. Srp Arh Celok Lek. 2012 Sep-Oct;140(9-10): 571-6.

29 Huang BT, Peng Y, Liu W, Zhang C, Huang FY, Wang PJ, et al. Increased interventricular septum wall thickness predicts all-cause death in patients with coronary artery disease. Intern Med J. 2015 Mar;45(3):275-83.

30 Graham-Brown MP, March DS, Churchward DR, Stensel DJ, Singh A, Arnold R, et al. Novel cardiac nuclear magnetic resonance method for noninvasive assessment of myocardial fibrosis in hemodialysis patients. Kidney Int. 2016 Oct;90(4):835-44.

31 Cai H, Lu R, Zhang M, Pang H, Zhu M, Zhang W, et al. Serum soluble Klotho level is associated with abdominal aortic calcification in patients on maintenance hemodialysis. Blood Purif. 2015;40(2):120-6.

32 Zheng S, Zheng Y, Jin L, Zhou Z, Li Z. Relationship between serum soluble Klotho protein and coronary artery calcification and prognosis in patients on maintenance hemodialysis. Iran J Public Health. 2018r Apr;47(4): 510-8.

33 Park CW, Shin YS, Kim CM, Lee SY, Yu SE, Kim SY, et al. Increased C-reactive protein following hemodialysis predicts cardiac hypertrophy in chronic hemodialysis patients. Am J Kidney Dis. 2002 Dec;40(6):1230-9.

34 Kim BS, Jeon DS, Shin MJ, Kim YO, Song HC, Lee SH, et al. Persistent elevation of C-reactive protein may predict cardiac hypertrophy and dysfunction in patients maintained on hemodialysis. Am J Nephrol. 2005 May-Jun; 25(3):189-95.

35 Maekawa Y, Ishikawa K, Yasuda O, Oguro R, Hanasaki H, Kida I, et al. Klotho suppresses TNF-alpha-induced expression of adhesion molecules in the endothelium and attenuates NF-kappaB activation. Endocrine. 2009 Jun;35(3):341-6.

36 Martín-Núñez E, Donate-Correa J, Muros-de-Fuentes M, Mora-Fernández C, Navarro-González JF. Implications of Klotho in vascular health and disease. World J Cardiol. 2014 Dec;6(12):1262-9. 